\title{
Family Planning Priorities for Women Living with HIV and Attitudes Towards Mandatory Family Planning Use While Taking Dolutegravir
}

\author{
Sarah Komanapalli, Julie G Thorne ${ }^{1}$, Caitlin Bernard ${ }^{2}$, John Humphrey ${ }^{3}$, Mercy Maina ${ }^{4}$, \\ Beatrice Jakait ${ }^{4}$, Rena Patel ${ }^{5}$ \\ 1 Department of Obstetrics and Gynecology, University of Toronto, Canada \\ 2 Department of Obstetrics and Gynecology, Indiana University School of Medicine, IN, USA \\ 3 Department of Medicine, Indiana University School of Medicine, Indianapolis, IN, USA \\ 4 Department of Pharmacy, Academic Model Providing Access to Healthcare (AMPATH), \\ Eldoret, Kenya \\ 5 Department of Medicine, Washington University, Seattle, WA, USA
}

Dolutegravir (DTG) is currently the first and second line antiretroviral therapy (ART) for women living with HIV (WLHIV). However, in 2018 Botswana Harvard AIDS Institute Partnership found that DTG exposure at conception corresponded with an increase in neural tube defects. Therefore, it is important that providers work with WLHIV to ensure they are aware of how to avoid unplanned pregnancies while on DTG. Telephone interviews were conducted with women living with HIV ages 21-59. The interviews were conducted in western Kenya, at clinical sites of the Academic Model Providing Access to Healthcare (AMPATH) program. Nineteen interview transcripts were coded using NVIVO 12. Domains included family planning use and shared decision making (SDM). Within these domains, three themes were used for this analysis: 1) tensions in ARV SDM and pregnancy, 2) decision making in family planning, and 3) decision making in family planning with DTG. The following three themes were discussed frequently: 1) When making decisions surrounding family planning, women preferred in person counseling and prioritized knowing the side effects of contraceptive methods; 2) WLHIV were often aware of the risk of HIV vertical transmission and valued preventing birth defects if told a medication may cause them; 3 ) When asked about using family planning while on DTG, WLHIV saw the benefit of preventing pregnancies. However, they did not believe family planning should be mandatory and preferred having a wide variety of contraceptive options. Understanding what factors influence women's family planning choices may improve SDM between patients and providers. This is especially important when it comes to preventing pregnancies in women taking DTG. WLHIV often understood the importance of effective ARTs and wanted to prevent birth defects. Therefore, it is important to navigate SDM conversations such that WLHIV are given control of their own health decisions. 